vol. $27-n^{\circ} 2$ | 2011

Numéro ouvert

\title{
Les musulmans en Italie entre crise identitaire et réponses islamistes
}

The Muslims in Italy between Identity Crisis and Islamist Answers

Los musulmanes en Italia entre crisis de identidad et respuestas islamistas

\section{Bartolomeo Conti}

\section{(2) OpenEdition}

Journals

\section{Édition électronique}

URL : https://journals.openedition.org/remi/5501

DOI : 10.4000/remi.5501

ISSN : $1777-5418$

\section{Éditeur}

Université de Poitiers

Édition imprimée

Date de publication : 1 octobre 2011

Pagination : 183-201

ISBN : 979-10-90426-01-6

ISSN : 0765-0752

\section{Référence électronique}

Bartolomeo Conti, «Les musulmans en Italie entre crise identitaire et réponses islamistes », Revue européenne des migrations internationales [En ligne], vol. 27 - n² | 2011, mis en ligne le 01 octobre

2014, consulté le 14 avril 2022. URL : http://journals.openedition.org/remi/5501 ; DOI : https://doi.org/ 10.4000/remi.5501 


\section{Note de recherche}

\section{Les musulmans en Italie entre crise identitaire et réponses islamistes}

\section{Bartolomeo CONTI ${ }^{1}$}

Ein 2009, le nombre des musulmans présents en Italie a presque atteint le
chiffre de 1,5 million qui, comparé aux 50000 du début des années 1980 , montre à quel point la présence de l'islam en Italie est récente ${ }^{2}$. À la différence d'autres pays où les musulmans se sont installés depuis plusieurs générations et où l'islam fait depuis longtemps partie intégrante du tissu social national, l'Italie n'a que récemment découvert que les musulmans étaient là pour rester et non pour partir le lendemain (Allievi, 2005). Cette découverte a eu lieu non seulement en raison du nombre croissant d'immigrés de religion musulmane qui participaient à la vie sociale et économique du pays, mais aussi à cause de l'émergence de l'islam organisé dans l'espace public. Ainsi, ce n'est qu'à partir des années 1990 que l'islam et les musulmans ont attiré l'attention des sciences sociales ; les recherches réalisées ${ }^{3}$, bien que peu nombreuses, ont cependant eu le mérite de montrer que la majorité des musulmans présents en Italie participent à des processus de sécularisation et de privatisation de l'expérience religieuse, comme on a pu l'observer dans d'autres pays européens à l'immigration plus ancienne. Aux côtés de cette majorité, il y a une minorité qui, par contre, refuse de cantonner l'islam à la vie privée et qui revendique sa présence dans l'espace public ou, autrement dit, qui reconstruit son identité et sa présence publique à partir de l'adhésion à l'islam. Cette minorité de musulmans, qui sera l'objet de cet article, a réussi à affirmer sa prééminence dans l'espace public italien grâce, d'un côté, à l'affirmation dans le champ religieux musulman d'un leadership que l'on pourra, selon le cas, nommer « islamiste » ou « fondamentaliste » et, de l'autre, à la

1 Doctorant au CADIS - EHESS, 190-198, avenue de France, 75244 Paris ; bartolomeo.conti@ ehess.fr

2 Par le terme "musulmans », on finit par entendre non seulement ceux qui sont des religieux pratiquants, mais aussi tous ceux qui ont dans l'islam un point de référence identitaire accepté consciemment ou qui leur est attribué. Sur l'utilisation de la catégorie «musulmans » en tant que catégorie néo-ethnique voir Roy (2002 : 65). Pour les chiffres et la composition concernant la présence de musulmans en Italie, voir le Dossier Statistique sur l'Immigration 2010, Spreafico (2005) et le site du CESNUR (www.cesnur.org/religioni_italia/islam.htm).

3 Parmi les études empiriques les plus significatives, on peut se référer au Dossier Statistique sur l'Immigration publié annuellement par Caritas-Migrantes ; l'enquête sur les musulmans de Vénétie (Saint-Blancat, 1999) ; l'enquête nationale faite en 2000 (Allam et Gritti, 2001). Pour une synthèse des résultats des études faits, voir Spreafico (2005). 
capacité de ce leadership à répondre à la demande de sens/identité exprimée par une partie des immigrés musulmans qui habitent en Italie. Une fois rapidement décrites les formes prises par la présence islamique, cet article abordera la crise identitaire d'une partie des immigrés musulmans et les réponses apportées par les différents courants de l'islam public qui opèrent sur le territoire italien ${ }^{4}$.

\section{L'ISLAM EN ITALIE}

À la fin des années 1970, après une longue histoire d'émigration, l'Italie commence à devenir un pays d'immigration, à l'image des pays nord-européens. Mais il s'agit d'un mouvement migratoire bien différent de celui qui avait caractérisé les pays du nord de l'Europe, non seulement parce qu'il coïncide avec le déclin de la grande industrie en tant que facteur d'activation de la demande de main-d'œuvre, mais aussi parce que la nouvelle immigration est plutôt constituée d'une population jeune, urbaine et alphabétisée, tandis que celle des cycles précédents était plutôt rurale et analphabète ${ }^{5}$. Outre ces deux éléments, qui concernent l'immigration en général, d'autres touchent plus spécifiquement aux immigrés de religion musulmane et contribuent à différencier l'islam italien de celui des autres grands pays européens :

\section{La diversification des pays d'origine}

Par rapport à des pays comme la France, l'Angleterre ou l'Allemagne, où la communauté musulmane est dominée par une ou deux nationalités, l'Italie présente une grande différentiation ethno-nationale. En particulier, il n'y a pas en Italie de population majoritaire qui soit capable de monopoliser le champ de l'imaginaire ${ }^{6}$. La fragmentation ethnonationale relève d'une part de l'emplacement géographique de l'Italie, avec ses deux façades méditerranéennes (balkanique et arabo-africaine) et d'autre part elle tient au fait que l'Italie n'a jamais maintenu de relations privilégiées avec ses ex-colonies, avec pour conséquence le faible nombre d'immigrés qui parlaient italien avant leur migration ou qui connaissaient la culture et les traditions italiennes.

\section{La rapidité d'entrée et d'insertion}

L'immigration musulmane en Italie est un phénomène récent, qui a été fortement influencé par deux facteurs extérieurs : d'un côté la présence d'un islam européen déjà structuré, de l'autre les dynamiques intérieures aux pays d'origine des immigrés, notamment l'importance nouvelle de la dimension religieuse dans la construction de l'espace public et dans la définition de l'identité individuelle. Ces deux facteurs exogènes ont, d'une certaine

4 Cet article se base principalement sur une enquête de terrain menée entre 2008 et 2010, au cours de laquelle ont été réalisés vingt-deux entretiens avec des leaders de l'islam en Italie.

5 Selon un sondage réalisé en 2000 (Allam et Gritti, 2001), le degré d'instruction des immigrés musulmans va de moyen à élevé ( 28,4 \% ont un diplôme universitaire, 44,3 \% un diplôme d'école supérieure, alors que les analphabètes représentent seulement 2,7\%).

6 Selon le Dossier Statistique sur 1'Immigration 2010, la plus grande communauté nationale « musulmane » est la communauté albanaise avec 441396 unités (même si, selon les études, seuls $70 \%$ des Albanais peuvent être définis comme musulmans), suivie de la communauté marocaine avec 431529 unités, de la tunisienne avec 103678 et de l'égyptienne avec 82064. 
manière, fait que l'islam italien a brûlé les étapes, à la différence de ce qui s'est passé dans les pays européens d'immigration ancienne 7 . La rapidité avec lequel l'islam s'est organisé pour revendiquer une place sur la scène publique italienne, en acquérant un rôle important dans les processus de socialisation des immigrés, a limité le développement de l'associationnisme laïque (ethnique ou culturel).

\section{La prédominance d'un islam de première génération}

L'islam italien reste essentiellement un islam de première génération. Avec la nouvelle génération, on observe cependant l'éloignement progressif d'un « islam ethnique », c'està-dire d'un islam qui a tendance à reproduire les façons de vivre et les codes de comportement des pays d'origine des immigrés et l'affirmation d'une logique individuelle dans le rapport au religieux (Frisina, 2007 ; Maddanu, 2009).

\section{La centralité des mosquées}

Bien qu'une petite partie seulement des musulmans fréquente régulièrement les mosquées, celles-ci jouent cependant un rôle primordial dans la représentation de l'islam en Italie ${ }^{8}$. Selon un rapport publié par le ministère de l'Intérieur, en mai 2007 les lieux de culte étaient au nombre de $735^{\circ}$. Il s'agit pour la plupart de locaux (souvent ex-magasins ou même caves) qui ont été transformés en salles de prière afin de répondre à la demande d'un nombre croissant d'immigrés musulmans. C'est précisément autour de la précarité de ces lieux de culte et la demande de nouveaux locaux qu'émerge le débat sur la place et la visibilité de l'islam dans l'espace public (Allievi, 2009). La « question des mosquées » se pose dans les villes, mais aussi dans les villages italiens avec en définitive la même conclusion : l'impossibilité de mettre en œuvre tous les projets de construction, souvent pour des raisons qui tiennent à l'ordre publique ou à l'impossibilité de modifier le statut légal d'utilisation des biens immobiliers.

7 À ce sujet, il est important de mentionner le concept de cycle migratoire, introduit par Bastenier et Dassetto, qui « vise à identifier les moments stratégiques du processus d'interaction conflictuelle et à l'issue incertaine qui s'engage dans l'espace public entre les "établis" et les "entrants" d'une société donnée » (1993: 10). Le concept de cycle migratoire désigne donc « l'ensemble du processus par lequel des populations qui appartiennent à un espace économique périphérique entrent, s'établissent et s'implantent dans un État-nation qui appartient aux pôles centraux de l'économie capitaliste » (Ibid. : 234).

8 Les chiffres relatifs aux musulmans qui fréquentent les mosquées sont très variables, également en raison du fait que beaucoup de petites salles de prière échappent à l'investigation sociologique. Guolo estime que $5 \%$ des musulmans fréquentent les mosquées (2005: 640). Selon le sondage fait en Vénétie, $74,2 \%$ des musulmans se définissent comme des croyants, et $38 \%$ du total affirment être pratiquants. $81 \%$ disent observer le jeûne du ramadan, $48 \%$ faire les cinq prières quotidiennes et $15 \%$ aller à la mosquée pour la prière du vendredi. Ces dernières données n'indiquent pas forcément des processus de sécularisation, car en Italie, la pratique religieuse peut être gênée par des difficultés d'ordre pratique, comme la difficulté de se rendre sur le lieu de culte ou le temps « chrétien » du calendrier du travail.

9 Il faut préciser que l'on se borne ici à appeler «mosquées » tous les lieux de culte islamiques, qui incluent les véritables mosquées, les centres culturels, les salles de prières et même les caves ou les garages transformés en lieux de rencontre et de prière pour les musulmans. 


\section{Les organisations islamiques en Italie}

L'islam en Italie est un islam pluriel, où cohabitent différentes façons de vivre la religion. Cette pluralité a été favorisée par la différentiation régionale et la parcellisation socio-économique qui caractérisent le contexte national italien (Saint-Blancat, 2004). L'expression « islam dialectal » est parfois utilisée pour décrire la spécificité de l'islam italien dans le panorama européen. Ainsi que plusieurs enquêtes l'ont montré, qu'ils soient sécularisés, traditionalistes ou le fruit d'un mélange religieux hétéroclite, les musulmans dans leur grande majorité en Italie vivent leur foi individuellement, préoccupés plutôt par la libre pratique de leur religion et la possibilité de la transmettre à leurs enfants ${ }^{10}$. Il existe aussi une minorité active, constituée d'organisations, de groupes, de mosquées et de salons de prière, qui refuse la dimension privatisée de la religion et qui revendique une présence islamique sur la scène publique.

La plus importante organisation islamique italienne, par le nombre de ses adhérents, les mosquées qu'elle gère et pour l'influence qu'elle exerce dans la définition de l'islam italien est sans doute l'Union des Communautés et des Organisations Islamiques en Italie (UCOII) ${ }^{11}$. L'islam de l'UCOII est aussi défini comme " l'islam des mosquées », notamment pour témoigner qu'une bonne partie des lieux de culte islamiques est affiliée à cette organisation, dans laquelle, pourtant, cohabitent plusieurs attitudes et tendances politico-religieuses. Sa direction est constituée principalement d'Italiens convertis et d'exétudiants moyen-orientaux, arrivés en Italie entre les années 1970 et le début des années 1990, souvent après avoir participé aux contestations islamistes dans leurs pays d'origine. L'UCOII adhère à la Fédération des Organisations Islamiques d'Europe, considérée comme la branche européenne du mouvement politico-religieux des Frères Musulmans.

Formé en 1966, le Centre Culturel Islamique d'Italie est l'association la plus ancienne. Elle représente le soi-disant « islam des États » de par ses liens avec plusieurs États musulmans sunnites, notamment l'Arabie Saoudite et le Maroc. Le Centre Culturel Islamique d'Italie gère la Grande Mosquée de Rome, la plus grande d'Europe. Elle a été inaugurée en 1995 et construite grâce au financement de l'Arabie Saoudite et du Maroc, qui se disputent le contrôle du Centre depuis sa création. C'est notamment le Maroc qui, afin de garder un contrôle sur ses propres expatriés, incite le Centre à jouer un rôle primordial dans la construction de la Fédération de l'islam italien, qui se présente comme une alternative à $1^{\prime} \mathrm{UCOII}^{12}$.

10 Plusieurs typologies ont été élaborées afin de décrire les différentes manières d' « être musulman » en Italie. Entre autres, soulignons celles de Saint-Blancat (1999) ; Pace et Perocco (2000) ; Gritti (2001).

$11 \mathrm{Au}$ cours de notre recherche de terrain, nous avons réalisé huit entretiens avec des leaders de centres islamiques affiliés à l'UCOII (www.islam-UCOII.it). Selon les propos de l'un d'eux : «l'UCOII fonctionne comme un syndicat, où chaque centre maintient une véritable autonomie ». Nous avons aussi interviewé le nouveau président de l'organisation et l'imam de Florence, le Palestinien Elzir Izzedin, lequel a affirmé que l'UCOII est : " une organisation apolitique et autonome. Et quand t'es autonome, on ne peut pas te faire du chantage et donc la seule manière, c'est de te détruire, parce que si on ne peut pas te faire du chantage, t'es dangereux ».

$12 \mathrm{Au}$ cours de notre travail de terrain, nous avons réalisé sept entretiens avec des leaders de mosquées qui adhérent à la Fédération pour l'islam italien. 
La Communauté Religieuse Islamique (www.coreis.it) est l'expression d'un petit groupe de convertis italiens, qui s'inspirent du soufisme de la confrérie Ahmadiya Idrissiya et de l'ésotérisme islamique de René Guénon. La COREIS, dont font partie des personnes de niveau culturel et social élevé est très active sur la scène publique italienne. Elle n'a cependant aucune implantation parmi les immigrés musulmans. Par contre, la COREIS revendique la représentation de l'islam italien, en promouvant un islam « totalement compatible avec la société italienne », qui s'oppose à ce qu'elle nomme " la dérive idéologique fondamentaliste » de l'UCOII.

La Jama'a al Tabligh est un mouvement islamique qui aujourd'hui prend de l'importance en Italie, aussi bien chez les immigrés que chez les Italiens convertis à l'islam. Bien qu'aucune étude n'ait été réalisée en Italie sur ce mouvement, les rares travaux concernant les musulmans réalisés dernièrement au niveau local témoignent de la pénétration graduelle des Tabligh auprès d'une partie importante des musulmans qui vivent en Italie $^{13}$.

Enfin, on trouve aussi ceux que l'on appelle ou qui se définissent comme « salafites ». Malgré le fait que cette appellation soit devenue un outil pour discréditer et délégitimer ses propres ennemis, le salafisme est un mouvement complexe, qui ne peut pas être réduit à sa branche violente. En Italie, le salafisme est bien présent et se manifeste parfois de manière transversale dans les différents centres islamiques éparpillés sur le territoire italien ${ }^{14}$. En Italie, le salafisme se manifeste parfois dans les différents centres islamiques éparpillés sur le territoire italien ${ }^{15}$. Le discours tenu par les salafites interviewés est clairement fondamentaliste et vise à une certaine séparation d'avec la société italienne, mais n'est pas nécessairement politique ou violent.

Les organisations que nous venons de citer ne constituent pas l'intégralité de l'islam organisé. Il en existe d'autres, qui sont généralement de petite taille ou de faible poids sociopolitique ou qui constituent des communautés séparées du reste des musulmans, comme c'est le cas par exemple de la confrérie sénégalaise des mourides.

\section{La rivalité pour la représentation de l'islam}

La lutte pour la représentation de l'islam en Italie est particulièrement vivace alors même qu'une minorité seulement de musulmans adhère à l'idée de l'islam public. Les rivalités sont amplifiées en l'absence d'autorités religieuses qui sont en situation de revendiquer une certaine légitimité et peuvent se présenter comme une référence pour les divers acteurs de l'islam italien. Cependant, il ne s'agit pas seulement d'une question

13 On se réfère notamment à une étude réalisée à Rome par Alessandra Caragiuli (à paraître). Au cours de notre recherche, nous avons réalisé des entretiens avec deux leaders de l'islam qui se réclamaient de ce mouvement.

14 À propos de la complexité du salafisme, voir Roy (2002); Amghar (2006) et Burgat (2007a).

15 L'exemple le plus important du salafisme est l'Institut Culturel Islamique, qui a son siège Via Jenner à Milan. Pour longtemps il a été la plus importante expression publique d'un islam qui refusait toute forme d'intégration, mais au cours des dernières années sa position a évolué et aujourd'hui il joue un rôle important non seulement dans les processus de socialisation des immigrés musulmans, mais aussi dans leur intégration dans la société italienne. 
de représentation : se joue d'une part le contrôle d'importantes ressources économiques, sociales et politiques et d'autre part, le pouvoir de définition, production et reproduction de la symbolique et de la gestion des lieux : mosquées, associations communautaires, Web. Selon Renzo Guolo, le conflit « a comme objet la définition des symboles, des systèmes de significations et de leurs limites, l'interprétation des croyances et de la tradition religieuse qui caractérise l'expérience de l'islam italien » (2005: 634).

Jusqu'à présent, toutes les tentatives de donner une représentation aux musulmans ont échoué. La dernière en date, et sûrement la plus importante, s'est faite en 2005, avec la création auprès du ministère des Affaires Intérieures de la Consulta pour l'islam italien. Nommée par le ministre dans la perspective d'arriver à former un islam italien, la Consulta a vite été bloquée par le conflit entre l'UCOII et la Grande Mosquée de Rome. La dernière étape de cette lutte, qui est bien présente dans les discours des leaders interviewés, a été la création en 2010 de la Fédération de l'islam italien, promue en particulier par la Grande Mosquée de Rome afin de créer une alternative crédible à l'UCOII.

\section{LES ISLAMISMES}

Avant de rentrer plus spécifiquement dans l'analyse de l'islam public en Italie il s'agit de clarifier ce qu'implique le terme controversé et trompeur d' " islamisme ». Tout d'abord, comme le soulignent plusieurs chercheurs (Lamchichi, 2001 ; Cesari, 2004 ; Roy, 2002), il faut distinguer l'islamisme du fondamentalisme (Pace, 2005). Selon Lamchichi, même si « les deux courants invitent les musulmans à opérer un "retour" et un "ressourcement" dans l'histoire islamique et les textes sacrés » (2001:25), « le fondamentalisme désigne une attitude religieuse qui consiste à s'en tenir rigoureusement aux textes sacrés » (Ibid. : 24), tandis que l'islamisme indique aussi (et plutôt) des courants politico-sociaux qui visent à la conquête du pouvoir afin d'islamiser la société et d'appliquer la charia. Mais la signification du terme " islamisme » ne fait pas l'unanimité chez les chercheurs, notamment parce qu'au fil du temps, ce terme a fini par indiquer une telle variété d'acteurs qu'il n'arrive simplement plus à tous les « contenir ${ }^{16}$. Souvent interprété comme une réponse à l'échec des politiques de modernisations économiques et sociales entreprises par les nationalistes arabes (Roy, 2002 ; Pace, 2005 ; Guolo, 2005 ; Burgat, 2007a), l'islamisme a en effet été décrit comme une idéologie politique qui vise à fonder la société autour de la religion et utilise celle-ci comme instrument de mobilisation politique pour accéder au pouvoir. Cependant, comme le souligne entre autres Göle dans la phase actuelle de l'islamisme, nommée « post-islamisme », « la ferveur révolutionnaire décline, le concert idéologique laisse la place à une multiplicité de voix, et prend forme un processus de distanciation et d'individualisation par rapport à une militance collective $[. .$.$] nous observons une transformation graduelle de ces mouvements d'une position$ politique radicale à une orientation plus sociale et culturelle [...]. Mais une orientation plus culturelle ne signifie pas une moindre orientation politique » (2006:5). L'islamisme couvre donc un vaste spectre de mouvements et d'organisations souvent très différents

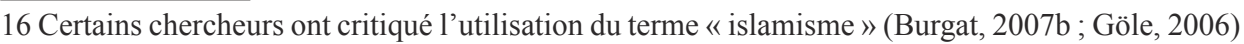
et d'autres ont forgé de nouveaux termes, tels que « postislamisme » ou « néofondamentalisme », pour décrire des mouvements en évolution et de plus en plus complexes (Roy, 2002 ; Lamchichi, 2001 ; Khosrokhavar, 2006). 
entre eux, mais qui partagent un discours politique basé sur l'islam ou, mieux encore, l'idée que l'islam ne doit pas être cantonné à la sphère privée, mais « occuper » l'espace public. C'est notamment là où l'islam est minoritaire, comme en Europe, que les discours et les actions des mouvements islamistes ont pris des formes encore plus variées.

Si donc le débat autour de l'islamisme est ample et varié, de la même manière que les définitions qui en ont été données, l'on souhaite ici souligner la position de ceux qui voient dans les mouvements islamistes non tant un retour en arrière des sociétés musulmanes, mais aussi une manière différente d'accéder à la modernité. En ce sens, si Burgat voit dans les mouvements islamistes la dernière étape du processus d'affranchissement des pays musulmans du contrôle occidental (2007a), Göle écrit qu' « islamisme est le nom de ce mouvement social à travers lequel un sentir partagé du déclin historique de l'Islam et de la stigmatisation de l'Islam par la venue des modernes définitions de soi et de la civilisation est transformé d'un déficit public en un avantage sub-culturel» (2006 : 15).

\section{CRISE ET RECOMPOSITION IDENTITAIRE DE L'IMMIGRÉ MUSULMAN}

L'arrivée et l'installation dans un autre pays impliquent une transformation de l'identité de chaque migrant (Basternier et Dassetto, 1993 ; Sayad, 1999). En ce qui concerne plus spécifiquement la recomposition identitaire des migrants musulmans, trois éléments sont à prendre en considération. Le premier est l'absence d'évidence sociale de la religion islamique, qui, de religion dominante telle qu'elle l'était dans les pays d'origine, devient religion minoritaire. Le passage en Italie, comme dans chaque autre pays non musulman, oblige le migrant à s'interroger sur le sens de son appartenance religieuse, c'est-à-dire sur ce que veut dire « être musulman » et, éventuellement, sur la façon de l'être. L'islam doit donc être revécu et repensé, c'est-à-dire devenir objet de réflexion. Le second réside dans le processus d'individualisation. La société d'accueil, ainsi que les lieux de socialisation communautaires, mettent sans arrêt l'immigré musulman face à la possibilité de choisir où, et avec qui, être. En d'autres termes, dans l'expérience migratoire, l'immigré musulman a la possibilité/nécessité de choisir quelle importance réserver aux différentes dimensions de son identité (religieuse, nationale, ethnique, idéologique, ou encore sociale, etc.) : « la reconstruction du lien identitaire se fait à partir de choix personnels, en favorisant ainsi le passage de la dimension communautaire, typique de l'islam, à la dimension individuelle. L'adhésion à l'islam doit être reformulée et devenir expérience subjective. Il s'agit d'un parcours où l'individu transite d'un héritage culturel défini pour toujours à la naissance, à une dimension volontaire, subjective, d'adhésion personnelle » (Guolo, $2005: 633)^{17}$.

Comme dans les autres pays d'Europe, en Italie le glissement progressif de l'expérience religieuse vers le subjectif a donné des résultats différents, parfois opposés. La

17 Pour la plupart des musulmans interrogés dans l'enquête menée en Vénétie, c'est au Coran d'assurer une solide référence pour la foi, plutôt qu'à une communauté structurée. La priorité donnée au Coran par les musulmans qui vivent dans un pays non musulman s'inscrit dans un processus d'individualisation de l'expérience religieuse, d'intériorisation de la foi qui est l'expression d'un individualisme propre à la modernisation. 
majorité des musulmans ont embrassé un islam sécularisé, c'est-à-dire qu'ils ont placé " la référence religieuse islamique dans la dimension subjective et privée, dans le vécu religieux intime » (Pacini, 2005: 16). L'autre résultat possible est le processus de réislamisation qui peut donner naissance à plusieurs formes d'islam orthodoxe, pas obligatoirement islamistes.

Enfin un troisième élément, découlant en grande partie des deux premiers, est la fragilité de l'immigré souvent exposé à de fortes pressions psychologiques, dues d'un côté à la perte de l'évidence sociale, et de l'autre, à l'absence d'institutions, telles que la famille ou les autorités religieuses traditionnelles, capables d'orienter ses choix. À cette fragilité psychologique, il faut ajouter une fragilité sociale de la plupart des immigrés qui, à leur arrivée en Europe, n'ont ni logement ni travail. Soumis à de nombreuses formes d'exploitation, ils vivent bien souvent en situation d'irrégularité qui rend leur existence et les contacts avec la société d'accueil, encore plus précaires. En outre, ils sont souvent objet de rejet ou de discrimination de la part des citoyens italiens, ainsi que des immigrés plus intégrés ${ }^{18}$.

Ces trois aspects sont fondamentaux pour comprendre les rôles que peuvent jouer les communautés auxquelles l'immigré musulman est susceptible d'appartenir, à commencer par la communauté islamique. C'est notamment dans les centres de socialisation islamistes que l'immigré musulman peut retrouver un langage, des rituels corporels et des pratiques qui contrastent avec la solitude à laquelle il peut être confronté, ainsi qu'avec la perte d'estime de soi que peut provoquer la confrontation avec la modernité occidentale. Comme l'ont montré des études réalisées dans d'autres contextes européens (Roy, 2002 ; Césari, 2004 ; Göle et Ammann, 2006 ; Khosrokhavar, 2006), en faisant de l'adhésion à l'islam un choix personnel plutôt qu'un héritage, les mouvements islamistes sont capables de renverser le sens de la subordination, de la perte de dignité et de l'honneur de l'être musulman, afin d'en faire une source de dignité et de supériorité exprimée individuellement et collectivement dans l'espace public. Comme l'affirme Göle, « le mouvement islamiste renverse la "différence in-désirée" d'être musulman en une adoption volontaire d'un symbole stigmatisant qui est ouvertement revendiqué et offensivement communiqué en public » (2006 : 16). En créant des espaces alternatifs distincts de l'espace public, ainsi qu'un langage et des symboles spécifiquement islamiques, les centres islamistes proposent aux musulmans en quête de repères et d'estime de soi une identité forte et revendiquée.

\section{LES RÉPONSES ISLAMISTES EN ITALIE}

Pour définir le panorama de l'islam public en Italie, les sciences sociales ont utilisé deux critères généraux : la relation avec la société italienne, c'est-à-dire le type et le niveau d'intégration souhaité, et la signification accordée au jihad, c'est-à-dire la place qu'occupe l'utilisation de la violence dans les relations politico-sociales. À partir de ces deux critères, nous pouvons décrire le mouvement islamiste, selon un schéma qui, en Italie, a notamment été introduit par Guolo (2005), entre néo-traditionalistes (activistes et

18 Sur l'exclusion des migrants en Italie, voir Dal Lago (1999). À propos de l'image des musulmans dans les médias italiens, voir Conte (2009). 
séparatistes) et radicaux : si les premiers sont les agents d'une intégration communautaire dans la société italienne et accordent au jihad une signification défensive, les seconds refusent toute forme d'intégration et entendent le jihad dans sa signification offensive, comme la lutte contre tous les incroyants. Évidemment, comme l'ont démontré les entretiens réalisés, cette schématisation n'est pas stricte, mais doit être lue en respectant l'ambivalence qui caractérise les individus et les organisations elles-mêmes (Tabboni, 1997).

\section{Islamisation et communautarisation néo-traditionalistes}

Si dans les pays musulmans les néo-traditionalistes visent à réislamiser des sociétés qu'ils jugent désormais largement sécularisées, en remettant la religion au centre de la sphère publique et en la transformant en facteur principal de régulation politique et sociale, là où l'islam est minoritaire, le mouvement néo-traditionaliste est porteur de deux aspirations principales : la réislamisation des immigrés musulmans et leur encadrement dans un sens communautaire. En Italie, l'islamisme néo-traditionaliste est principalement représenté, mais pas exclusivement, par l'UCOII et constitue le type d'islam organisé le plus répandu sur le territoire ${ }^{19}$. Pour une partie des musulmans, c'est donc dans les mosquées et les centres culturels qui font partie de l'UCOII que la demande de sens/ identité trouve une réponse. Dans ces lieux, l'environnement, les actions et le discours tenu visent non seulement à combattre les processus de sécularisation et de privatisation de la foi qui, comme on l'a vu, touchent une bonne partie des musulmans en Italie, mais aussi à canaliser la crise identitaire des immigrés afin de faire de la religion le facteur intégrant de la vie individuelle et sociale. Selon la vision néo-traditionaliste, l'identité islamique doit se manifester à travers un style de vie et des comportements qui distinguent le « bon » musulman du reste de la société italienne. Cela peut se manifester dans l'aspect extérieur (le voile, la barbe ou la Jalabbiah), la pratique religieuse (les prières), le respect de la distinction pur/impur (à partir des obligations alimentaires ou de la séparation entre hommes et femmes), l'étude du Coran, la familiarité avec une certaine littérature politico-religieuse et la participation à la vie communautaire. Une des parties intégrantes du processus de réislamisation des musulmans est la réduction, ou même l'élimination, de tout ce qu'on ne peut ramener au religieux, c'est-à-dire des autres facteurs intégrateurs ou de socialisation comme la nationalité, l'ethnie, l'idéologie, la classe sociale, etc. Il s'agit de réduire toutes les autres facettes de l'immigré musulman afin d'en consacrer une seule fondatrice de la nouvelle identité développée pendant l'expérience migratoire, l'identité

19 L'affirmation de cette minorité dans le champ musulman a été favorisée par plusieurs facteurs, comme l'absence d'autorités islamiques traditionnelles, la faiblesse de l'associationnisme laïque et ethnique, la petite représentativité des autres organisations islamiques et enfin par le fait que la quasi-totalité des musulmans sont arrivés en Italie au moment où les pays islamiques étaient déjà pris par un important retour à la mosquée, qui a souvent fini par se prolonger en Italie. Voir Guolo (2004 et 2005). 
islamique ${ }^{20}$. Comme l'affirme le président d'un centre affilié à l'UCOII :

"Notre travail est celui de réveiller l'identité musulmane, l'identité religieuse en tant qu'appartenance à une identité culturelle... Nous essayons de faire connaître cette identité qui offre tant de solutions aux problèmes de l'humanité, aussi bien pour les individus que pour les pays. Il y a des solutions pour tous les problèmes, à tous les niveaux de la vie humaine, au niveau économique, politique et social».

Dans le contexte migratoire, la reconstruction de « l'individu islamique » est strictement liée à la formation d'espaces culturellement distincts du reste de la société, basés sur la dichotomie pur/impur. Comme l'affirme Guolo, le « fondement de l'action néo-traditionaliste en Italie, en plus de la $d a$ 'wa, l'appel à l'islam, est donc l'hijra, la rupture et la séparation d'avec la "communauté impure". L'hijra néo-traditionaliste est mise en œuvre à travers un processus de communautarisation, c'est-à-dire à travers la construction sociale d'une communauté qui, plus qu'à l'intégration individuelle de ses membres, vise à négocier sur une base collective un statut de dérogation de citoyenneté, statut qui définisse le degré d'auto-exclusion nécessaire à la reproduction de sa propre séparation » $(2004: 9)^{21}$.

Le processus de communautarisation, en plus d'une mémoire, d'un langage et d'un univers symbolique spécifiquement islamique, prend plusieurs formes concrètes. D'abord la création d'espaces communautaires, séparés du reste de la société italienne, comme les centres culturels, les mosquées, les écoles coraniques, les librairies et les bibliothèques islamiques. Il s'agit de contre-espaces qui revitalisent le sens de l'appartenance communautaire, soit à travers des discours (mémoire du pays de provenance, mémoire d'immigration, récits religieux, etc.), soit à travers des actes performatifs (vestimentaires, alimentaires, corporels, temporels, etc.) ou rituels (prières, mariages, etc.). Comme l'affirme Göle, " à l'intérieur de ces espaces semi-publics, non pas ouverts à tous, mais seulement à ceux qui gardent un sens spécifique d'appartenance à l'islam, le soi musulman est façonné et mis en œuvre quotidiennement, en renversant le stigmate islamique en une source de confiance en soi et d'autonomisation à travers un engagement en conflit avec les modèles dominants de la société » (2006 : 34). Il faut ajouter les organisations d'encadrement, comme les organisations juvéniles et féminines, telles que l'Association des femmes musulmanes en Italie - la branche féminine de l'UCOII, qui compte parmi ses objectifs la sauvegarde de l'identité de la femme islamique en Italie ou l'Association des Jeunes Musulmans d'Italie, qui, par contre, affirme de plus en plus l'exigence de se distinguer de « l'islam des pères » (Frisina, 2007 ; Maddanu, 2009). Sur un plan plus politico-social, la communautarisation des musulmans s'exprime principa-

20 Le processus de déculturation (décrit par Roy, 2002 et Guolo, 2005) est rempli d'obstacles, non seulement à cause de la fragmentation et de la compétition dans le champ religieux, mais surtout à cause du fait que, pour la plupart des musulmans, la religion ne semble pas être l'élément le plus important dans leur référence identitaire. En effet, selon le sondage national réalisé en 2000, seulement $12,9 \%$ des interviewés pensent que leur identité réside dans leur foi religieuse, alors que 42,8 \% l'identifient d'abord avec l'appartenance nationale et 40,1\% soutiennent avoir une identité différente de l'identité religieuse ou nationale.

$21 \mathrm{Au}$ cours des entretiens, nous avons pu constater comment plusieurs leaders néo-traditionalistes aspirent à des lois spécifiques pour les musulmans, notamment en ce qui concerne le droit de la famille. 
lement à travers le rôle que l'UCOII essaie de se donner, notamment au niveau local, en se présentant comme l'unique médiateur entre les institutions et les musulmans, même en ce qui concerne les questions non strictement religieuses. Comme le disent Pace et Perocco, « en prenant des charges publiques et en occupant des positions avec ses propres adhérents dans les administrations locales (bureaux pour étrangers, médiateurs culturels, etc.) et dans les associations sectorielles (syndicats, secteur tertiaire), ou en jouant le rôle de référents et porte-parole des populations musulmanes immigrées sur des thématiques différentes (cours d'alphabétisation, besoins socio-sanitaires, cours professionnels, insertion au travail) les mosquées ouvertes par l'UCOII se proposent de plier les projets d'intervention sociale, les politiques locales pour les immigrés, à la perspective globale du vécu de la foi » (2000: 16). Ici réside l'une des sources des forts contrastes qui opposent l'UCOII aux représentants des États nationaux, notamment ceux du Maroc, qui essayent de garder le contrôle des leurs citoyens en insistant sur leur alliance nationale plutôt que sur leur appartenance religieuse ou idéologique.

Au cœur des processus de socialisation de l'UCOII se trouve aussi une vision des relations internationales, axée sur la légitimité de la lutte, même violente, de certains peuples contre l'occupation et l'oppression, comme dans le cas des Palestiniens, des musulmans de Bosnie, des Irakiens ou des Afghans. Ces orientations sont exprimées sur la scène publique à travers déclarations et manifestations qui ont souvent été la cible de fortes critiques de la part des autres organisations islamiques en Italie, ou même des institutions $^{22}$. Dans les centres de socialisation islamiques, par contre, se manifestent des formes de solidarité qui, dans le passé, se sont traduites par un soutien matériel apporté aux musulmans de Bosnie pendant la guerre, et qui continuent actuellement avec les Palestiniens.

Les lieux de la déconstruction identitaire de l'immigré musulman et de sa redéfinition dans un sens islamique sont d'abord les mosquées, c'est-à-dire les lieux de visibilité de l'islam dans l'espace public. L'importance de la mosquée est bien synthétisée dans les propos de deux leaders islamiques interviewés :

"La mosquée, depuis que le Prophète a fondé la première mosquée de l'histoire, est au centre de toute l'activité sociale, de tous ses aspects. Et c'est lié à la vision

islamique du monde, de la vie, parce que l'islam gère tous les aspects de la vie, aussi les choses quotidiennes, les plus petites choses de notre vie... La mosquée est donc un point de référence pour tous les aspects de la vie ».

" J'ai commencé à pratiquer avant d'arriver en Italie, parce que j'avais peur de me perdre. Il y a cette peur et donc on se lie à ces centres, afin de se maintenir. C'est pour ça qu'on est venus dans ces centres... Quand je suis arrivé, ce n'était pas facile, avec toutes ces femmes, avec toute cette liberté. Ce n'était pas facile, mais le centre m'a beaucoup aidé à me maintenir et à m'intégrer petit à petit ».

22 Le cas qui a soulevé le plus de polémiques a été celui du manifeste de l'UCOII, publié en août 2006 dans plusieurs journaux nationaux, intitulé « Ieri stragi naziste-oggi stragi israeliane 》, où les massacres perpétrés par Israël étaient comparés à ceux des nazis. À ce sujet, voir aussi le controversé document de condamnation du terrorisme international, présenté par l'UCOII à la suite des attentats de Londres : www.islam-UCOII.it/NOTERRORISMO.htm. 
La tâche de "former » la communauté des croyants n'est généralement pas confiée à des imams issus des institutions religieuses traditionnelles, mais plutôt à des imams qui se sont formés dans le cadre de la politisation de la militance religieuse, dont le discours n'est pas seulement religieux, mais aussi politique et social. Venant souvent d'un cercle d'émigrés qui ressentent le besoin de sauvegarder les traditions d'origine, ils ne sont pas le plus souvent des experts ou des chefs communautaires, mais plutôt des acteurs qui savent répondre aux questions des fidèles en se présentant comme les garants des idées traditionnelles sur la femme, la famille, l'éducation, la société. Comme nous avons pu le constater au cours de notre recherche, la quasi-totalité des imams que l'on a interviewés sont des autodidactes qui, comme nous a dit le président d'un petit centre islamique, " font ce qu'ils n'auraient jamais eu ni le droit ni la possibilité de faire dans leur pays : guider la communauté».

Les propos qui suivent sont un exemple de la façon dont s'est formée la quasitotalité des imams en Italie :

"Quand j'étais au Maroc, j'étais attaché à la religion et j'allais écouter les leçons, apprendre la religion chez certains maîtres qui enseignaient dans les mosquées. J'ai ainsi acquis mon premier enseignement de la religion. Et ensuite, j'ai continué à étudier $»$.

Le rôle qu'ils jouent est bien synthétisé par Saint-Blancat, qui écrit que les imams en Italie « doivent répondre soit aux besoins socio-économiques liés au processus d'immigration soit aux demandes religieuses. Dans beaucoup de cas, ils font encore les deux. Ils ont le rôle traditionnel de l'imam : conduire la prière, assurer le sermon du vendredi $(k h u t b a)$, enseigner les principes coraniques aux jeunes, présider aux rites de passage et fournir des conseils juridiques dans la mesure de leurs propres compétences. Ils sont aussi continuellement sollicités pour résoudre des problèmes contingents et d'ordre social, comme les permis de séjour, les problèmes liés au logement, les droits au welfare (notamment la santé publique) ou pour donner des conseils sur le marché du travail ou sur comment se débrouiller dans la byzantine législation italienne. La liste est sans fin » (2004:3).

Les lieux néo-traditionalistes de l'UCOII se présentent donc aux musulmans comme les principales forteresses d'une identité perçue comme menacée ; des lieux qui peuvent répondre à leur demande de sens, grâce à un contexte spatial et symbolique centré sur « l'être islamique », sur l'appartenance à la oumma, sur la spécificité de l'identité islamique, porteuse de styles de vie et de systèmes de valeurs différents de ceux proposés par la société italienne. Selon deux imams interviewés :

"J'aime une société liée à la foi, parce que la foi éduque la personne à bien se comporter, à respecter les autres. La foi, c'est la meilleure manière de construire une personnalité équilibrée ».

«Le musulman pratiquant doit être le meilleur comme citoyen parce que la religion qu'il pratique tous les jours l'empêche de voler et en général de faire des choses mauvaises. Donc, si tu es un bon citoyen, tu dois être aussi un bon musulman, 
En ce sens, nous avons pu confirmer ce qu'avaient souligné Pace et Perocco. Pour ces auteurs, l'islam peut être un véritable facteur d'intégration « parce qu'il aide l'individu dans le processus d'insertion sociale, à travers le sens de l'appartenance à une communauté de foi et de styles de vie éthiquement orientés » (2000:7). Les nombreuses activités qui se déroulent généralement dans les centres islamiques de l'UCOII, entre autres, et qui vont des cours d'arabe ou religieux pour les enfants aux cours d'italien pour les immigrés et les femmes, de l'aide sociale aux familles en difficulté aux activités ludiques et récréatives, constituent autant d'initiatives qui contribuent aussi bien à la communautarisation qu'au processus d'insertion sociale.

C'est notamment au niveau local que les centres islamiques gérés par l'UCOII participent aux processus d'intégration, soit à travers la promotion de comportements éthiquement orientés qui empêchent des formes de déviance, soit à travers une participation à la vie sociale qui, au cours des dernières années, est devenue de plus en plus intense. Cela se manifeste par des collaborations, soit avec les institutions locales, comme l'administration, les écoles ou même les prisons, soit avec la société civile, comme les rencontres interreligieuses. Beaucoup de ces centres affiliés à l'UCOII se sont, cette dernière décennie, implantés dans les centres urbains et se sont organisés en lieux d'éducation éthique. Selon le secrétaire national de l'UCOII et imam de Florence :

« Nous croyons que la mosquée doit être la mosquée de la ville, et pas seulement de la communauté islamique. Avec cette vision, nous avons une ouverture vers ceux qui ne sont pas musulmans... Et si la mosquée doit être de la ville, elle ne peut pas être en dehors de la ville ».

Si l'UCOII représente la branche activiste du néo-traditionalisme, selon la catégorisation de Guolo, d'autres centres néo-traditionalistes sont qualifiés de « séparatistes », afin de souligner la rupture sociale et culturelle avec la société italienne. En refusant toute forme d'intégration, qu'elle soit individuelle ou communautaire, ces centres optent pour la construction d'espaces islamiques qui puissent créer une sorte de société parallèle, où le seul facteur d'intégration est représenté exclusivement par l'adhésion à la religion islamique et donc, par l'appartenance à la oumma, la communauté des croyants : c'est seulement en suivant les prescriptions des textes que le musulman peut rester « pur parmi les impurs ». Comme l'on a pu le constater au cours de notre recherche, les leaders interviewés qu'on peut qualifier de « séparatistes », comme les salafites ou les Tabligh, n'ont que peu d'intérêt à faire partie de la société italienne, qu'ils analysent et jugent à travers la grille pur/impur :

«L'islam est une protection pour les personnes. Dans une société où il y a l'islam, tu ne peux pas voir un gars bourré, un voleur, ou quelque chose de mauvais. Il y aura toujours du respect... On peut donner l'exemple de quelqu'un qui rentre à la maison le soir avec une bonne odeur par rapport à quelqu'un qui sent le vin et dit des gros mots. L'islam veut que tu sois toujours propre, au niveau du cerveau, des habits, comme au niveau du cour».

"Quand une femme est couverte, elle est respectée... Tu la regardes comme une sœur. Quand, par contre, tu vois une femme nue, comment tu la regardes? Quand tu vois une femme couverte, tu as un grand respect, pour l'autre non. Tu peux la regarder de la tête aux pieds ». 
C'est aussi par rapport au jihad que certains centres séparatistes se distinguent de l'UCOII et, plus généralement, des autres centres néo-traditionalistes ${ }^{23}$. Bien que les attitudes puissent être différentes même à l'intérieur d'un seul centre, les séparatistes élargissent la définition du jihad défensif pour englober diverses réalités selon une vision assez manichéenne des relations internationales, qui part de l'idée que l'islam doit se défendre puisqu'il est attaqué partout dans le monde.

"Tu peux bien voir que le monde est contre l'islam. Si tu regardes ce qui s'est passé dans la bande de Gaza ou ce qui se passe dans le monde : ils attaquent toujours l'islam ».

"Quand on fait une guerre dans le monde, on la fait pour deux raisons : pour la richesse, le pétrole, ou pour faire la guerre à l'islam ».

Le jihad, dans sa version défensive, est considéré comme légitime, soit contre toute forme de néo-colonialisme et d'oppression occidentale, soit contre les gouvernements musulmans « impies » qui ont mis l'islam sous le joug de l'Occident.

\section{Leaders, discours et socialisation de l'islamisme radical}

Dans les pays musulmans, l'islamisme radical théorise un modèle d'action sociale qui prévoit l'islamisation « d'en haut », c'est-à-dire par la conquête du pouvoir. Dans le contexte italien, où l'islam est minoritaire, l'action de l'islamisme radical a été plutôt dirigée vers les pays islamiques, là où règnent des régimes considérés comme apostats et impies, et qu'il faut renverser. Comme l'ont démontré plusieurs rapports de magistrats, l'Italie a été un lieu d'organisation des réseaux de soutien logistique, de propagande, de financement, de recrutement de volontaires à envoyer sur les champs de bataille ${ }^{24}$. Pendant la guerre des Balkans, le territoire italien a servi de base aux mujahidin et aux combattants en route vers la Bosnie : l'Italie « était le point de passage privilégié pour la Bosnie, elle a été pour les islamistes ce que le Pakistan a été pour l'Afghanistan » (Spataro, 2004). L'autre objectif des islamistes radicaux est la conquête d'espaces de production du savoir religieux et symbolique à l'intérieur de la communauté musulmane italienne ; ils s'opposent à ceux des musulmans qu'ils considèrent comme moralement corrompus ou apostats, à commencer par les adhérents à l'islamisme néo-traditionaliste, notamment de l'UCOII, accusés de compromission avec les institutions italiennes et d'avoir abandonné la défense $\mathrm{du} \ll$ vrai islam ».

23 On fera la distinction entre islamisme et fondamentalisme : certains centres salafites ou Tabligh, peuvent être définis comme fondamentalistes parce qu'ils font une lecture des textes extrêmement stricte, en essayant de conformer leurs conduites à celles que l'on prête au Prophète et aux premiers califes. Par contre, leur discours ne veut pas investir l'espace public et n'aborde que marginalement les questions internationales.

24 Une description des réseaux radicaux a été donnée par le procureur de Milan, Armando Spataro (2004) : les groupes radicaux, qui en Italie se sont spécialisés notamment dans la fabrication de faux documents, ont longtemps conservé leur identité ethno-nationale, mais semblent désormais dépassés par le jihadisme global. 
Par la nature même de son discours et de son action, l'islamisme radical opère à la frontière de la légalité et donc en dehors de l'espace public, échappant ainsi à l'investigation sociologique et devenant objet quasi exclusif d'enquêtes journalistiques ou judiciaires. Il existe toutefois quelques mosquées ou centres islamiques, où le discours d'imams radicaux a circulé plus explicitement. Il s'agit notamment de l'Institut islamique de Viale Jenner ${ }^{25}$ et de la mosquée de la Via Quaranta à Milan, de quelques mosquées dans la région de Turin et d'autres éparpillées sur le territoire italien. C'est donc dans ces lieux que l'on a pu retracer le parcours de certains leaders radicaux, écouter leurs discours, et enfin avancer des hypothèses sur la façon dont ils arrivent à répondre à la demande de sens/identité exprimée par une partie des musulmans présents en Italie ${ }^{26}$.

Une première catégorie de leaders que l'on pourrait qualifier de « transnationaux » est constituée de ceux qui sont arrivés en Italie après avoir participé aux luttes qui ont opposé les islamistes aux pouvoirs en place dans des pays musulmans, comme l'Algérie ou l'Égypte. Leur discours est centré sur le jihad, qui assume ici un sens politique de lutte à mener contre les «tyrans » des pays musulmans et ceux qui les maintiennent au pouvoir, notamment les pays occidentaux. La légitimité dont ces leaders jouissent dérive de leur passé de combattants, de leur participation aux réseaux jihadistes internationaux ainsi que de leur combat pour l'affirmation de l'islam dans le monde. Parfois soupçonnés de gérer d'importantes ressources financières, leur attitude est de ne pas se mêler à la société italienne afin de préserver une identité islamique " pure », en vue d'un retour dans le Dar al islam. C'est, entre autres, le cas d'Abu Imad, imam de l'Institut Islamique de Viale Jenner, condamné en 2008 pour terrorisme international, qui s'est formé en Égypte, où il a fait partie de la Jama'a Islamiyya et où il a été accusé d'avoir pris part à l'assassinat de Sadate. C'est aussi le cas de l'Égyptien Abou Omar, imam de la mosquée de Via Quaranta, kidnappé à Milan le 17 février 2003 par la CIA et transféré en Égypte. Lié à la Jama'a Islamiyya et ensuite à Ansar al Islam, Abou Omar est un exemple typique de radical transnational ${ }^{27}$.

Un deuxième groupe de leaders radicaux est constitué par ceux qu'il est possible de définir comme des « autodidactes », qui se sont affirmés après leur arrivée en Italie,

$25 \mathrm{Au}$ sein de l'Institut ont transité des représentants de formations radicales, surtout égyptiennes, qui y ont aussi occupé des positions dirigeantes. Entre autres, on peut rappeler que l'émir de l'Institut, Anwar Shaban, est mort dans un attentat en Bosnie. D'autres affiliés de l'Institut ont été arrêtés en 1995, accusés d'avoir envoyé des armes et des volontaires pour la guerre en Bosnie ; en 2008, plusieurs personnes liées à l'Institut, y compris l'imam, ont été condamnées pour terrorisme international. À ces accusations l'Institut a toujours répliqué que beaucoup de gens passent chez eux et qu'il est impossible de tous les contrôler : voir à ce sujet l'interview du président de l'Institut (Paci, 2006 : 64). L'on doit aussi ajouter qu'au cours des dernières années, l'Institut a assumé des positions beaucoup moins conflictuelles que dans le passé, comme le démontrent certaines formes de collaboration avec les institutions et la société civile de Milan et ainsi que cela nous a été confirmé par le procureur de Milan Spataro lors d'un entretien réalisé en 2010.

26 Pour cela, nous nous baserons aussi sur des études réalisées dans d'autres contextes nationaux, comme celles de Khosrokhavar (2006) et Martinez (2008).

27 Pour un panorama des réseaux radicaux qui ont transité entre la mosquée de Viale Jenner et celle de Via Quaranta à Milan, voir la reconstruction faite par le tribunal de Milan dans son mandat d'arrestation de Abou Omar, où sont rapportés les dialogues entre militants enregistrés par la police italienne : www.statewatch.org/cia/documents/milan-tribunal-abu-omar.pdf. 
grâce notamment à l'absence d'autorités religieuses traditionnelles ${ }^{28}$. Ils se présentent comme des défenseurs de l'identité islamique, en tenant des discours sur la famille, sur le rôle de la femme, sur les relations entre croyants et incroyants, et sur la défense du « vrai islam » face à l'Occident « colonisateur et pervers ». Certains d'entre eux ont construit leur autorité au sein d'une communauté musulmane locale, en s'appuyant sur des moyens financiers propres qui leur ont permis de contrôler plusieurs lieux de rencontres et de socialisation. C'est le cas de Bouriqui Bouchta, qui s'était autoproclamé « imam de Turin ». Appelé l'« imam boucher » pour son rôle de premier plan dans l'abattage et la distribution de la viande halal, il a pu compter sur sa fortune et sur un discours de défense identitaire pour s'affirmer comme référence de l'islam turinois ${ }^{29}$.

Qu'ils soient autodidactes, issus d'un groupe de musulmans soucieux de préserver leur identité ou provenant des réseaux internationaux, les leaders de l'islamisme radical partagent avec une partie des islamistes néo-traditionalistes l'idée que l'islam est partout menacé, dans les pays musulmans comme dans les pays où les musulmans ne sont qu'une minorité. C'est donc aussi autour du jihad que se construit leur discours, comme le démontrent les propos de l'imam de Carmagnola, Fadlallah Mamour, qui a déclaré que : « le jihad est une obligation pour chaque musulman quand le territoire musulman est attaqué. C'est un devoir subjectif, fard kifaya, pour chaque prédicateur musulman d'inciter les fidèles à s'engager pour le jihad. Dans le cas spécifique de l'Afghanistan, s'agissant d'un territoire attaqué où la population musulmane ne peut pas se défendre elle-même, le jihad est un devoir objectif, fard ayn, auquel aucun fidèle ne peut échapper. L'Afghanistan était le seul pays musulman sur Terre... Moi, mon jihad, je le fais ici en Italie avec la $d a$ 'wa, l'appel à l'islam » (Allam, 2002). Ce discours se nourrit de l'identification avec des réalités emblématiques qui sont vécues avec une grande intensité par les membres de la communauté, à tel point que les souffrances subies par les " frères musulmans » en Palestine, Irak, Afghanistan ou Tchétchénie deviennent les souffrances de chaque musulman qui s'identifie à la oumma ${ }^{30}$.

La description de la société italienne comme culturellement raciste, sexuellement agressive, incroyante et oppressive est le deuxième pivot du discours des leaders radicaux, notamment de ceux qui se sont affirmés dans le contexte migratoire comme défenseurs d'une identité perçue comme menacée. Leur discours invite à un respect rigoureux de la pratique et des obligations religieuses, afin de renforcer des frontières symboliques qui seules peuvent défendre la communauté et protéger le « vrai croyant » des " tentations maléfiques » de la société occidentale : «ne pas se mêler » est donc à la base de leur relation avec la société italienne.

28 On peut citer en exemple la petite communauté musulmane de Ponte Fulcino, à côté de Pérouse, où en 2007 des personnes considérées comme intégrées, sans pratiquement jamais n'avoir eu de contacts avec des radicaux, ont essayé de constituer une petite cellule terroriste.

29 Bouchta a été expulsé en 2005 pour des « raisons d'ordre et de sécurité publique », après que les Américains en 2001 eurent arrêté en Afghanistan et transféré à Guantanamo un Marocain résidant à Turin, qui avait fréquenté une des ses mosquées, où il aurait été recruté pour aller combattre en Afghanistan.

30 Sur la portée de cette identification avec une oumma en lutte, voir Roy (2002) et Khosrokhavar (2006), ainsi que les affirmations emblématiques des leaders et des moudjahidin enregistrées dans les mosquées de Milan : www.statewatch.org/cia/documents/milan-tribunal-abu-omar.pdf. 
On peut donc avancer l'idée de l'existence de deux groupes principaux de musulmans radicaux en Italie : tout d'abord, ceux qui ont vécu la lutte contre les pouvoirs en place dans leur pays et qui continuent leur combat politico-religieux en Italie. Pour eux, il s'agirait de retrouver dans le discours radical une justification de leur propre vécu : combat armé, prison, torture, exil, etc. Ensuite, ceux qui n'ont pas trouvé une place dans le nouveau pays, et qui vivent en conflit avec une société « raciste et agressive » qui les a exclus et humiliés. Leur radicalisation s'inscrirait donc essentiellement à l'intérieur d'une forte revendication identitaire.

L'opposition entre le « vrai croyant » et l'incroyant, l'islam et l'Occident ${ }^{31}$, permet aux musulmans en « crise d'identité », ou « humiliés et vaincus », de trouver dans le code moral et l'identification à la oumma les ressources nécessaires à leur rédemption. Le jihad devient ainsi l'instrument pour échapper à la soumission, à la défaite, pour la délivrance individuelle et collective. De perdant le fidèle devient gagnant, de membre d'une culture inférieure et dominée il devient membre d'une communauté élue qui triomphera, d'humilié il devient un « pur supérieur aux impurs ». Une intense pratique religieuse et l'adhésion totale à la morale religieuse, lui garantiront le salut et une réalisation de soi autrement perçue comme impossible (Spataro, 2008). De manière beaucoup plus totalisante et conflictuelle que ceux qui adhèrent à l'islamisme néo-traditionaliste, le musulman radical reformule ainsi son identité dans un sens religieux, c'est-à-dire en poussant jusqu'à l'extrême le processus de déculturation : son identité est uniquement formée de son adhésion à l'islam.

\section{CONCLUSION}

L'islam italien de la fin des années 2000 n'est plus celui de la fin des années 1990 (Allievi, 2005). La plupart des centres islamiques, à commencer par ceux qui adhérent à l'UCOII, ne sont plus des lieux qui visent à créer une séparation nette par rapport au reste de la société italienne, mais ont plutôt commencé un parcours d'intégration ou même d'inclusion (Wieviorka, 2008). Les propos de l'un des imams de l'UCOII interviewés à Rome sont à ce titre exemplaires :

«Désormais, on ne peut plus parler de communauté islamique, parce que la communauté donne le sens de la non appartenance, donne le sens qu'un jour on repartira. Aujourd'hui, il faut plutôt parler de la minorité islamique, ce que nous sommes devenus, qui donne aussi le sens de l'appartenance au lieu, à la société dans laquelle on vit, même si on est d'une autre religion ».

31 «À l'Occident s'oppose... une communauté d'Allah refaite à l'image de la période héroïque de l'islam sous le Prophète... La néo-oumma se définit positivement là où elle trouve du négatif en Occident ou dans les pays musulmans soumis au règne de la jahiliya... La néo-oumma qu'ils appellent de leurs vœux est une société rangée où les "turpitudes" occidentales n'auraient pas droit de cité : la "nudité" de la femme (c'est-à-dire le fait qu'elle ne soit pas voilée), la promiscuité entre les sexes, la sortie de la femme hors du foyer en vue de travailler avec les hommes, l'égalité juridique entre les sexes, l'homosexualité, la consommation d'alcool, la liberté religieuse » (Khosrokhavar, $2006: 368$ ). 
À la base de cette transition, il y a en particulier une deuxième génération qui est en train de grandir et qui a finalement amené « les pères » venus d'ailleurs à la conclusion qu'il n'y aura pas de retour dans le dar-al-Islam. C'est notamment au niveau local que les centres islamiques, tout comme les musulmans en général, font de plus en plus partie de la société italienne. L'intégration est en effet à l'œuvre dans les écoles, sur les lieux de travail, dans les rapports avec les institutions et les autres acteurs sociaux, à commencer par les associations catholiques. Mais si l'intégration se fait au quotidien et au niveau local, la conflictualité et la séparation sont encore bien présentes lorsque le discours investit l'espace public transnational, soit le rapport entre islam et Occident, ou alors la lecture des textes religieux, qui continue en règle générale à rester plutôt fondamentaliste.

Nous conclurons en citant les propos du président du centre islamique de Sassuolo, qui s'exprime sur l'avenir de la nouvelle génération qui grandit aujourd'hui en Italie :

«Mon espoir, c'est que mon fils devienne un Italien musulman. Qu'il devienne quelqu'un qui connaît bien la situation ici, qui puisse aider, qui puisse devenir quelqu'un d'important à la mairie, mais qui en même temps puisse rester musulman. Que les gens voient qu'il est né ici, qu'il a grandi ici, et qu'en même temps, il n'ait pas perdu son identité et ne se soit pas éloigné de sa ville, Sassuolo. Ça, c'est mon rêve ».

\section{Références bibliographiques}

ALLAM Magdi (2002) Bin Laden in Italia. Viaggio nell'islam radicale, Milan, Mondadori, 215 p.

ALLAM Magdi e GRITTI Roberto (2001) Islam, Italia, Milan, Guerrini, 195 p.

ALLIEVI Stefano (2009) Conflicts over Mosques in Europe: Policy issues and trends, London, Alliance Publishing Trust.

ALLIEVI Stefano (2005) How the Immigrant has Become Muslim, Revue Européenne des Migrations Internationales, 21 (2), pp. 135-163.

AMGHAR Samir (2006) Le salafisme en Europe. La mouvance polymorphe d'une radicalisation, Politique étrangère, 2006/1, pp. 65-78.

BASTENIER Albert et DASSETTO Felice (1993) Immigration et espace public, Paris, L'Harmattan, $314 \mathrm{p}$.

BURGAT François (2007a) L'islamisme en face, Paris, La Découverte, 350 p.

BURGAT François (2007b) L'islam des uns et l'islamisme des autres [en ligne]. URL : http://halshs. archives-ouvertes.fr/docs/00/14/99/34/PDF/Francois_BURGAT_Islam_et_Islamisme.pdf

CESARI Jocelyne e PACINI Andrea (Eds.) (2005) Giovani musulmani d'Europa. Tipologie di appartenenza religiosa e dinamiche socio-culturali, Turin, Fond. Agnelli.

CESARI Jocelyne (2004) L'islam à l'épreuve de l'Occident, Paris, La Découverte, 276 p.

CONTE Daniela (2009) I musulmani di Italia: questi sconosciuti? L'immigrazione musulmana raccontata in TV, Ricerche di Pedagogia e Didattica, 4 (2), pp. 1-15 [en ligne]. URL : http://rpd. cib.unibo.it/article/view/1589/954

DAL LAGO Alessandro (1999) Non-persone: l'esclusione dei migranti in una società globale, Milan, Feltrinelli, 279 p.

FRISINA Annalisa (2007) Giovani musulmani d'Italia, Carocci, Roma.

GÖLE Nilüfer and AMMANN Ludwig (2006) Islam in public: Turkey, Iran and Europe, Istanbul, Istanbul Bilgi University Press, 543 p.

GUOLO Renzo (2004) Sociologia degli attori musulmani: leadership islamiste in Italia, Religioni e società: rivista di scienze sociali della religione, 50, pp. 8-12. 
GUOLO Renzo (2005) II campo religioso musulmano in Italia, Rassegna Italiana di Sociologia, 4, pp. 631-657.

KHOSROKHAVAR Farhad (2006) Quand Al Qaida parle : témoignages derrière les barreaux, Paris, Grasset, 421 p.

LAMCHICHI Abderrahim (2001) L'islamisme politique, Paris, L'Harmattan, 174 p.

MADDANU Simone (2009) La deuxième génération de musulmans en Italie : nouvelles pratiques quotidiennes chez les jeunes de l'association GMI, Thèse, EHESS, Paris.

MARTINEZ Luis (2008) Structures, environnement et basculement dans le jihadisme, Cultures \& Conflits, 69, pp. 133-156.

PACE Enzo (2005) L'ideal-tipo fondamentalismo, Critica sociologica, 152, pp. 1-12.

PACE Enzo e PEROCCO Fabio (2000) L'Islam plurale degli immigrati in Italia, Studi Emigrazione/ Migration Studies, 137, pp. 2-20.

PACI Francesca (2006) Islam e violenza: parlano i musulmani italiani, Rome, Laterza, 173 p.

ROY Oliver (2002) L'islam mondialisé, Paris, Seuil, 208 p.

SAINT-BLANCAT Chantal (2004) Imam e responsabili musulmani: le modalità di interazione con la società locale, Congres L'Islam en Italie, Turin.

SAINT-BLANCAT Chantal (ed.) (1999), L'islam in Italia: una presenza plurale, Rome, Ed. Lavoro, $211 \mathrm{p}$.

SAYAD Abdelmalek (1999) La double absence. Des illusions de l'émigré aux souffrances de l'immigré, Paris, Seuil, 448 p.

SPATARO Armando (2008) Why Do People Become Terrorists? A Prosecutor's Experiences, Journal of International Criminal Justice, 6 (3), pp. 507-524

SPATARO Armando (2004) Il terrorismo islamico in Italia e nel mondo, Conseil Supérieur des Magistrats, Rome.

SPREAFICO Andrea (2005) La presenza islamica in Italia, Instrumenta, IX (25), pp. 173-243.

TABBONI Simonetta (1996) Le multiculturalisme et l'ambivalence de l'étranger, in Michel Wieviorka Éd., Une société fragmentée. Le multiculturalisme en débat, Paris, La Découverte, pp. 227-250.

WIEVIORKA Michel (2008) L'intégration : un concept en difficulté, Cahiers internationaux de sociologie, 125 (2), pp. 221-240. 